\title{
利用离散最优传输的纹理重整算法
}

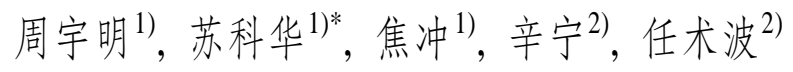 \\ 1) (武汉大学计算机学院 武汉 430072) \\ 2) (中国空间技术研究院通信与导航卫星总体部 北京 100094) \\ (skh@whu.edu.cn)
}

\begin{abstract}
摘 要: 随着建模与扫描技术的不断发展, 三维模型的精细程度不断提高, 场景数据量不断提升. 为了解决纹理碎 片化、纹理空间利用率过低的问题, 提出了一种基于离散最优传输的纹理重整算法. 给定一个带纹理的模型, 首先计 算其保形参数化映射; 接下来通过离散最优传输对保形参数化进行调整, 计算得到原始模型的保测参数化映射; 最 后根据网格的初始参数化与计算得到的保测参数化对原始纹理进行重新映射, 将数量众多的小纹理整合得到一个大 纹理. 为验证算法的有效性, 从 Sketchfab 上选择了不同种类与复杂度的扫描模型进行实验, 对整合前后的纹理空间利 用率、采样率和渲染图像的峰值信噪比进行了计算并与其他算法进行了对比. 实验结果表明, 所提算法的空间利用率能 够始终保持在 $100 \%$, 且纹理采样率分布平均, 同时渲染图像的峰值信噪比与其他方法不相上下, 均在 $35 \mathrm{~dB}$ 以上.
\end{abstract}

关键词: 离散最优传输; 网格参数化; 纹理重整

中图法分类号: TP391.41 DOI: 10.3724/SP.J.1089.2021.18632

\section{Texture Integrationvia Discrete Optimal Mass Transportation}

\author{
Zhou Yuming ${ }^{1)}$, Su Kehua ${ }^{1 *}$, Jiao Chong ${ }^{1)}$, Xin Ning ${ }^{2)}$, and Ren Shubo ${ }^{2)}$ \\ 1) (School of Computer Science, Wuhan University, Wuhan 430072) \\ ${ }^{2)}$ (Institute of Communication and Navigation Satellite, China Academy of Space Technology, Beijing 100094)
}

\begin{abstract}
Powered by modeling and scanning technology, the fineness of 3D models and the data size is increasing rapidly. To solve the problem of texture fragmentation and low texture space utilization, a texture integration approach based on optimal mass transportation is presented. Given a textured model, first the shape preserved parameterization is computed, then the discrete optimal mass transportation is used to adjust the shape preserved parameterization, obtaining the measure preserved parameterization. Finally, this parameterization and the initial parameterization are used to remap the original texture, integrating the small textures into a big texture. In the experiments, proposed approach is compared with other approaches in terms of texture space utilization, sampling rate, and peak signal to noise ratio of the rendering image on different types and complexity of scanned models on Sketchfab. The experimental results show that the texture space utilization can always be $100 \%$ and the sampling rate is uniformly distributed. Besides, the peak signal to noise ratio is comparable to other approaches, which are all above $35 \mathrm{~dB}$.
\end{abstract}

Key words: discrete optimal mass transportation; mesh parameterization; texture integration

收稿日期: 2020-09-07; 修回日期: 2020-11-10. 基金项目: 国家重点研发计划(2018YFB2100500); 国家自然科学基金(61772379); 武大华为空间信息技术创新实验室资助项目(YBN2018095106). 周宇明(1995一), 男, 硕士研究生, 主要研究方向为计算机图形学; 苏科华 (1979一), 男, 博士, 副教授, 硕士生导师, CCF 会员, 论文通讯作者, 主要研究方向为计算共形几何、计算机视觉; 焦冲(1995一), 男, 硕士研究生, 主要研究方向为计算机图形学; 辛宁(1982一), 男, 博士, 高级工程师, 主要研究方向为三维几何处理; 任术波(1976一), 男, 博士, 工程师, 主要研究方向为三维几何处理. 
纹理映射是一种简单并高效的增加多边形网 格真实度的算法 ${ }^{[1]}$, 在不增加物体表面几何特征的 条件下，能有效地模拟物体表面的视觉特征，并在 极短的时间内制作出逼真的几何模型.

近年来，随着大规模三维重建技术的快速发 展，尤其是航空遥感、机载激光雷达及无人机倾斜 摄影测量等三维数据获取手段的广泛普及，城市 级乃至更大区域的真实三维场景数据的快速获取 已不再困难，这为基于真实三维场景的应用带来 了极大的便利. 但是, 这也使模型与纹理的数据量 急剧增加. 通过扫描得到的模型纹理往往是由数 量众多的小纹理组成的, 这些小纹理在渲染时通 常被合并为一个图集统一载人显存中. 由于单个 纹理数据量小，因此对单个纹理进行压缩或简化 的意义并不大. 另外, 单个纹理的边界往往是不规 则的，合并后的图集中往往存在空白区域，这些空 白区域同样需要占用显存与带宽, 造成了资源的 浪费. 现代图形处理器都提供了专用的高速缓存 以加速纹理数据的存取, 进而提高整个图形系统的 运行效率. 但是由于其价格昂贵, 高速缓存的容量 往往都非常有限, 特别是在一般的商用图形处理器 上. 对纹理数据进行压缩并使用不同的调度算法控 制纹理数据的加载与释放是一种常见的解决方案.

为了解决纹理碎片化、有效纹理占比过低的问 题, 本文提出了一种基于离散最优传输的纹理重 整算法. 对于给定的带碎片纹理以及原始参数化 映射的三角形网格, 本文首先计算其保形参数化 映射, 之后使用基于离散最优传输的保测参数化 算法对该保形参数化进行调整，使得到的参数化 网格能够尽可能地保持原始网格的特征. 最后根 据网格的原始参数化与计算得到的保测参数化 对原始纹理进行重新映射, 将碎片化的小纹理整 合得到一个大纹理.

纹理整合相关的工作大致可分为 2 类，一类是 通过在纹理空间上对纹理进行优化达到整合纹理 的目的，如通过对纹理进行频率分析直接对原始 纹理进行拉伸达到纹理的优化 ${ }^{[2]}$; 另一类是通过 优化小纹理的边界, 并在纹理空间上对每个小纹 理进行平移或旋转以调整其整体的排布达到纹理 整合的目的 ${ }^{[3-5]}$. 这类问题实质上是一个 NP 难问 题 ${ }^{6]}$. 本文算法通过计算参数化的方式构建原始纹 理与整合后纹理之间的映射，比其他算法更加简 洁直观，既考虑模型的面积畸变以及高斯曲率的 高低，也能够保证整合后纹理的空间利用率达到 $100 \%$, 具有重要的理论价值和现实意义.

\section{1 最优传输}

最优传输问题的目标是找到一个将物质从起 始区域移动到目标区域的映射, 该映射能够最小 化物质运输的代价, 而又保证物质的总量不变. 设 物质所在的区域为 $\Omega$, 从起始区域到目标区域的 映射为 $T: \Omega \rightarrow \Omega$, 从起始点 $x$ 到目标点 $T(x)$ 的运 输代价为 $c(x, T(x))$, 则传输代价最小的映射为

$$
T^{*}=\arg \min \int_{\Omega} c(x, T(x)) \mathrm{d} u(x) .
$$

物质的总量在映射前后不能发生变化, 对于 $\Omega$ 上的任意集合 $X \subset \Omega$, 设 $u(x)$ 和 $v(x)$ 为区域 $X$ 内起始区域和目标区域上某一点物质的量, 计算 最优传输的过程必须始终满足条件

$$
\forall X \subset \Omega, \int_{T^{-1}(X)} u(x) \mathrm{d} x=\int_{X} v(x) \mathrm{d} x
$$

通过式(1)可以看到, 最优传输问题的核心目 的是计算物质传输方案 $T: \Omega \rightarrow \Omega, T$ 将物质从起 始区域以一定的代价运输到目标区域，并保证运 输过程中的代价最小. 而式(1)保证了传输过程中 的物质不会凭空地增加或减少, 因此该条件称为 保持测度条件。

在计算最优传输的过程中，起始区域和目标 区域也可看做有限数量离散点所构成的集合. 这 时函数 $u(x)$ 和 $v(x)$ 的定义域不再是一个连续的区 域, 而是一个有限点集. 需要注意的一点是, 当函 数在离散区域上进行积分运算时, 有限位置上不 为 0 的点会被忽视, 积分的结果仍将为 0 . 为了解 决该问题, 可以使用概率测度对物质量的多少进 行表示. 对于目标区域中的每个离散点, 使用被运 输到目标区域物质量的多少为权重, 赋予该离散 点一个狄拉克测度. 这样, 最优传输问题就可以表 示为 $T^{*}=\inf _{T: X \rightarrow X} \int_{X} c(x, T(x)) \mathrm{d} \mu$. 此时, 式(1)可以 改写为

$$
v=T_{\#} \mu
$$

映射 $T$ 将概率测度 $\mu$ 映射为概率测度 $v$. 使用概 率测度对原有问题进行改写后, 对于 $\forall X \subset \Omega$, 式 (2)等价于 $T_{\#} \mu(X)=v(X)=\mu\left(T^{-1}(X)\right)$.

由于传输过程中需要始终满足保持测度条件, 并且最优传输映射是一个非对称映射, 即起始区 域和目标区域是不能够互换的，因此直接对最优 传输映射进行求解是非常困难的. 为解决这个难 题, Kantorovich ${ }^{[7]}$ 提出了松驰的概念. 对于 $X \times Y$ 上的联合测度 $\rho$ ，任何一个从 $\mu$ 到 $v$ 的映射都可 以表示为 $\rho(A \times Y)=\mu(A), \rho(X \times B)=\mu(B)$, 其中, 
$A$ 和 $B$ 为 $\Omega$ 上区域 $X$ 和 $Y$ 的子集. Kantorovich 通 过引人中间测度 $\rho$ 解决了最优传输无法直接求解 的问题. 随后, Brenier ${ }^{[8]}$ 给出了最优传输映射与凸 几何之间的内在联系, Villani ${ }^{[9]}$ 在 Brenier 工作的基 础之上进行了总结与推广. Brenier ${ }^{[8]}$ 指出, 存在一 个凸函数 $\varphi: x \mapsto \nabla u(x)$, 其梯度映射就是最优传输 映射. 该凸函数即为 Brenier 势能函数, 是一个由 若干超平面所组成的上包络. 如图 1 所示, 通过垂 直正交投影可以得到其在平面 $\Omega$ 上的胞腔分解. 这里, 目标测度被离散为有限点集 $\left\{\boldsymbol{p}_{i}\right\}_{i=1}^{k}, \boldsymbol{p}_{i}$ 为平 面 $\Pi_{i}$ 的梯度. 构成上包络的每个平面 $\pi_{i}$ 都被映射 为 $\Omega$ 上的胞腔 $W_{i}$, 故上包络对应的梯度映射为 $W_{i} \rightarrow \boldsymbol{p}_{i}$. 由于映射前后测度保持不变, 因此每个 胞腔 $W_{i}$ 的面积在单位化后应等于其目标测度.

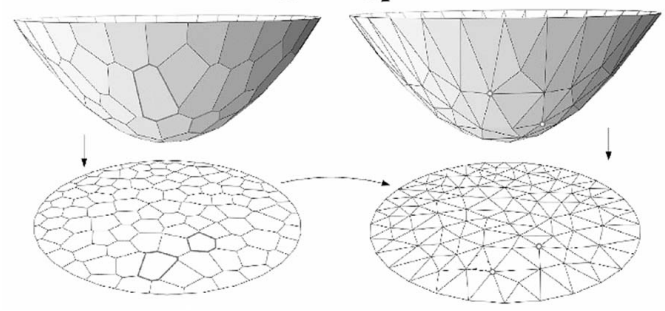

图 1 离散 Brenier 势能函数和最优传输映射

详细的理论基础与算法的实现细节可以参考 文献[10-12]，这 3 篇文献分别介绍了 3 种不同的基 于最优传输的参数化算法.

基于最优传输的保测参数化算法需要给定一 个初始映射以及每个顶点处的目标测度. 所谓保 测参数化, 即通过操控曲面的测度构造多种不同 的全局参数化以完成不同的任务. 如通过操控曲 率测度构造保曲率测度参数化完成离散采样, 通 过操控面元测度构造保面积参数化完成纹理重构. 通过将这两者组合在一起不仅能够使被整合纹理 的面积畸变非常小，也考虑了模型的几何形状，使 褶皱变化较大的地方能够获得更多的权重. 本文 根据原始模型上每一顶点处的 one-ring 面积以及 高斯曲率指定目标测度 $\gamma_{i}=\alpha \hat{K}_{i}+\beta \hat{A}_{i}$. 其中, $K_{i}$ 为顶点 $v_{i}$ 处的高斯曲率; $A_{i}$ 为顶点 $v_{i}$ 处的 one-ring 面积. 由于测度描述的是每个顶点上对应度量的 分布情况，并且曲率与面积的单位不同无法直接 相加, 故还需要将 $K_{i}$ 和 $A_{i}$ 单位化, 得到 $\hat{K}_{i}$ 和 $\hat{A}_{i}$. $\hat{K}_{i}$ 和 $\hat{A}_{i}$ 可以看做曲率和面积在三角网格上的分布, 并且它们量纲相同, 可以直接相加. $\alpha$ 和 $\beta$ 为曲率 与面积的权重系数, 可以根据实际情况进行调整,
实验中本文将 $\alpha$ 和 $\beta$ 均设置为 1. 根据计算可以得 到目标测度 $\gamma_{i}$, 最后将 $\gamma_{i}$ 也进行单位化, 即可得到 输人算法的目标测度 $\hat{\gamma}_{i}$. 根据给定测度的不同, 参 数化的结果也会不同. 如图 2 所示, 可以看到在初 始保形参数化以及不同目标测度下的保测参数化 中，弥勒佛头部的大小以及形状都各不相同.

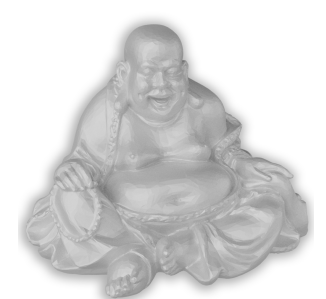

a. 原始模型

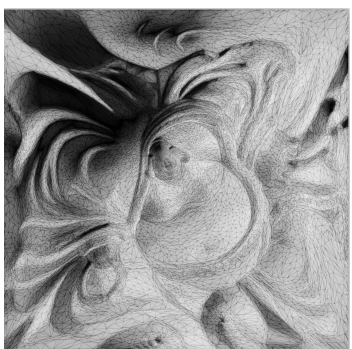

c. $\alpha=1, \beta=0$ 时 的保测参数化

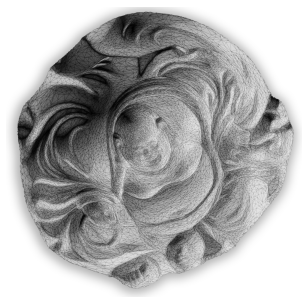

b. 初始保形参数化

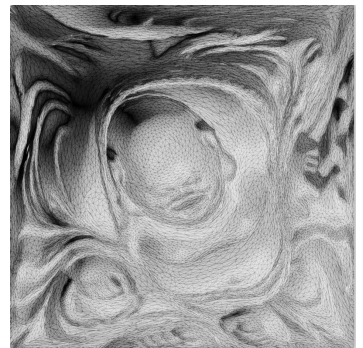

d. $\alpha=0, \beta=1$ 时 的保测参数化
图 2 保测参数化

通过最优传输计算保测参数化的整体过程如 图 3 所示. 首先计算最优传输的初始映射; 接下来 给定最优传输的目标测度; 最后在常曲率的目标 参数域上通过最优传输计算得到最终的保测参数 化映射.

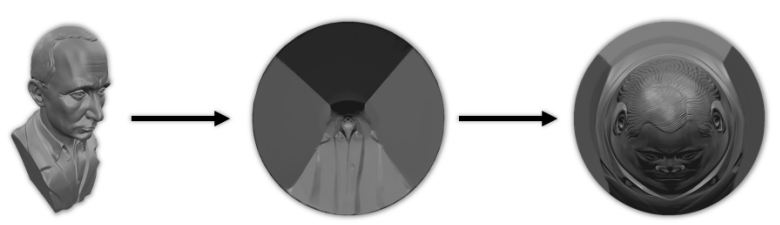

图 3 基于最优传输的保测参数化的整体计算过程

\section{2 纹理重整}

根据模型自带的初始参数化映射 $f$, 以及使 用最优传输算法计算得到的保测参数化映射 $g$, 可以构建映射 $f$ 与映射 $g$ 之间的变换关系. 利用 该变换关系可以达到对原有纹理进行重新整合的 目的.

网格中的每个三角形在模型自带的初始参数 
化映射与保测参数化映射后会发生不同的畸变, 这 2 个畸变后的三角形之间的变换关系可以用一 个仿射变换矩阵进行表示. 本文用 $\triangle p_{a} p_{b} p_{c}$ 和 $\triangle p_{a}^{\prime} p_{b}^{\prime} p_{c}^{\prime}$ 分别表示经过初始参数化映射和保测参 数化映射后的三角形, 如图 4 所示. 设 $\triangle p_{a} p_{b} p_{c}$ 和 $\triangle p_{a}^{\prime} p_{b}^{\prime} p_{c}^{\prime}$ 中的 3 个顶点的坐标分别为 $\left(x_{i}, y_{i}\right)$ 和 $\left(x_{i}^{\prime}, y_{i}^{\prime}\right)$, 其中, $i=1,2,3$. 设 $\boldsymbol{T}$ 为变换矩阵, 则有

$$
\left[\begin{array}{lll}
x_{1} & y_{1} & 1 \\
x_{2} & y_{2} & 1 \\
x_{3} & y_{3} & 1
\end{array}\right] \boldsymbol{T}=\left[\begin{array}{lll}
x_{1}^{\prime} & y_{1}^{\prime} & 1 \\
x_{2}^{\prime} & y_{2}^{\prime} & 1 \\
x_{3}^{\prime} & y_{3}^{\prime} & 1
\end{array}\right] .
$$

通过求解该线性方程即可得到三角形之间的变换 矩阵.

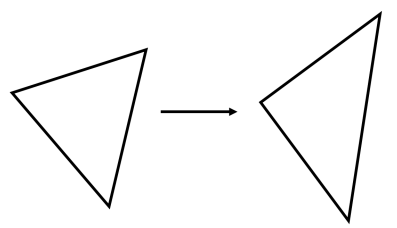

图 4 三角形之间仿射变换

对于保测参数域中的每个三角形，计算其二 维包围盒后; 遍历该包围盒对应区域在纹理中的 每一个像素点, 并使用 PNPoly 算法 ${ }^{[13]}$ 判断该像素 点的位置是否在三角形内部. 对于在三角形内部 的像素点, 首先使用变换矩阵 $\boldsymbol{T}^{-1}$ 计算出该像素变 换之前的位置坐标, 然后利用该坐标在原始纹理 上进行采样，即可得到当前遍历三角形内该点处 的颜色值. 遍历完成后即可得到如图 5 所示重整后 的纹理. 对于重整后的纹理还可以对其进行向下 重采样, 以减少贴图所占的内存空间. 从结果中可 以看出, 整合后的纹理完全避免了碎片化现象, 空 间利用率达到 100\%, 边界整齐, 不需要对接缝做 特别处理，这将极大地简化后续三维场景的优化 工作.

注意到这里的仿射变换矩阵 $\boldsymbol{T}$ 是逐三角形计 算, 虽然在三角面片相邻处会存在变换矩阵不连 续的问题，但是这并不会对纹理映射的结果产生 影响. 这是因为在图形处理器(graphics processing unit, GPU)上, 纹理映射操作也是逐三角面片进行 的. 在渲染的过程中，每个三角面片都会被光栅 化, 每个栅格处的属性值都是由对应三角形的 3 个 顶点插值得到的. 因此，逐三角面片地计算仿射变 换矩阵并不会影响纹理映射, 只需要在算法实现 时避免像素的重复运算即可.

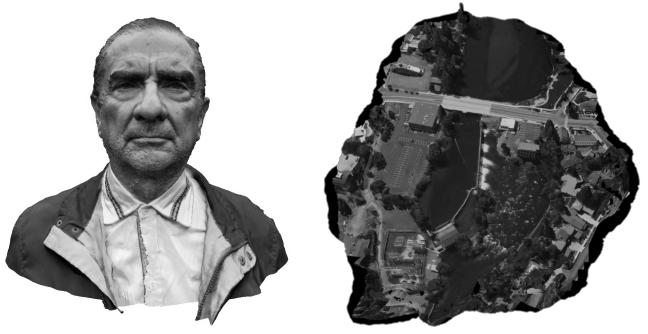

a. 原始模型

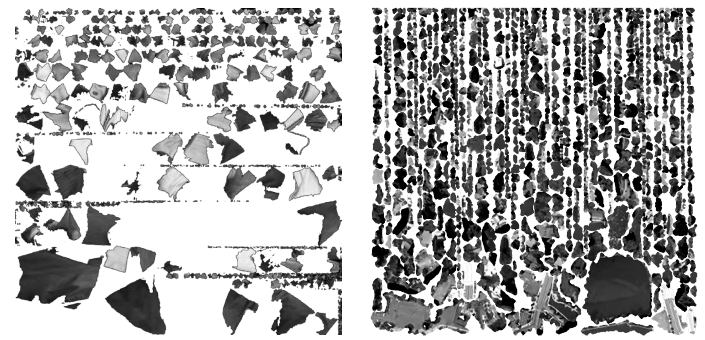

b. 重整前的纹理

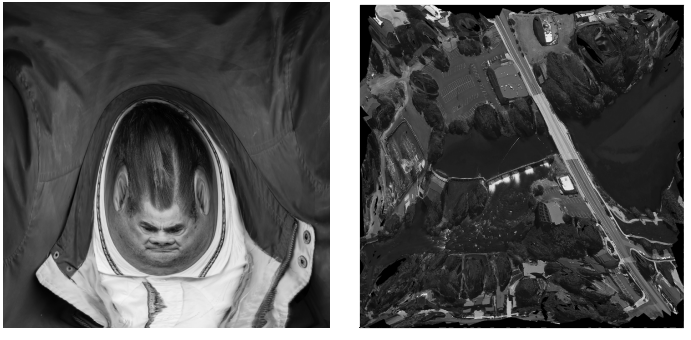

c. 重整后的纹理

图 5 重整前后的纹理对比

\section{3 模型预处理}

\section{1 带亏格模型剪开}

基于离散最优传输的保测参数化算法对于输 人的模型有以下几点要求.

（1）模型具有流形结构.

(2) 模型是单边界的.

(3) 模型的亏格数为 0 .

所以，需要对亏格大于 0 或边界数大于 1 的模 型进行相应的预处理. 本文使用曲面基本群的求 解算法火烧法 ${ }^{[14]}$ 将多亏格的三维模型剪开为 0 亏 格的单边界模型. 而对于非流形网格, 一般可以选 择拆边或拆点的方法进行处理.

火烧法的直观描述为: 给定曲面 $M$, 假想其 表面长满野草; 在曲面上任选一点 $\boldsymbol{p}$, 从 $\boldsymbol{p}$ 点开 始点燃曲面, 火源沿着曲面向四周蔓延, 若曲面存 在亏格, 则这 2 道火焰必然相交. 相交处熄灭形成 轨迹, 该轨迹称为割迹 $C$; 火焰烧过的区域称为 曲面的基本域. 因为每个基本域对应了一个拓扑 圆盘的区域, 所以沿着割迹 $C$ 对模型进行剪开, 得 到的网格应该是单边界且 0 亏格的. 火烧法的算法 
可描述如下.

输人. 曲面 $M$.

输出. 剪开后的三角形网格.

Step1. 对曲面 $M$ 进行三角剖分形成网格 $G$.

Step2. 任选网格 $G$ 的某个三角形面元 $f$ 作为起点, 广度优先遍历网格 $G$ 中的所有面元, 并将遍历过的面 元和面元包含的边进行标记.

Step3. 遍历结束后，网格中未被标记的边共同构 成了一棵树, 对树的分支进行剪裁, 留下的路径即为割 迹 $C$, 沿着 $C$ 将模型剪开即可得到目标网格。

如图 6 所示, 亏格为 1 的猫咪经过该算法得到 了一条割迹, 沿着该割迹剪开得到亏格为 0 的单边 界模型。该算法从理论上保证任意亏格的模型都 可被剪开.

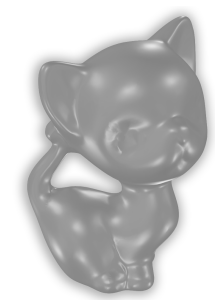

a. 猫咪

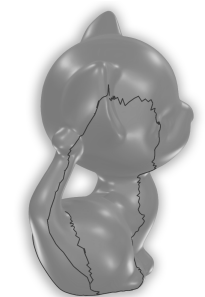

c. 割迹(侧视图)

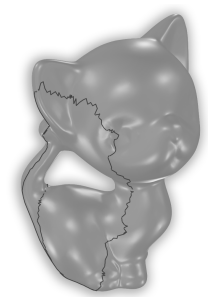

b. 割迹(正视图)

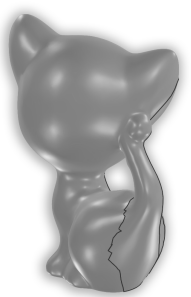

d. 割迹(背视图)
图 6 带割迹的猫咪

\section{2 保形参数化}

基于离散最优传输的保测参数化算法需要输 人一个带有初始映射的模型, 并且为了最大化重 整后纹理的空间利用率, 参数化的模型边界应为 矩形. 由于高质量的输人能够减少最优传输算法 优化迭代的次数, 故本文选择了能够保证模型的 局部形变尽可能小的保形参数化作为最优传输的 初始映射. 现有的保形参数化算法非常多, 如共形 映射 ${ }^{[15]}$ 、调和映射 ${ }^{[16]}$ 、Ricci 流 ${ }^{[17]}$ 和 Calabi 流 $^{[18]}$ 等. 本文实验中选择使用调和映射计算最优传输 的初始映射.

\section{4 本文算法步骤}

纹理重整算法的整体过程可描述如下.

输人. 带有三角剖分的流形网格 $M$, 网格对应 的初始纹理图片 $I$.
输出. 重整后的纹理图片 $I^{*}$.

Step1. 计算网格 $M$ 的亏格数 $g$.

Step2. 若 $g \geqslant 1$, 则使用火烧法将模型剪开.

Step3. 使用调和映射算法得到保形参数化.

Step4. 使用最优传输算法得到保测参数化.

Step5. 计算每个面元在模型自带的初始参数化映 射与保测参数化映射后 2 个三角形之间的仿射变换矩 阵, 并使用该矩阵对原始纹理进行重映射得到重整后的 纹理 $I^{*}$.

\section{5 实验结果及分析}

纹理重整的效果是本文探讨的重点, 这部分 从纹理的空间利用率以及纹理映射的质量 2 个方 面论证了基于最优传输的纹理重整算法的有效性. 本文使用的实验平台为 Windows 10 操作系统的台 式计算机, 配置为 i7-7700CPU, GTX 1080Ti GPU, 16 GB RAM; 使用的三角网格处理库为 OpenMesh 6.3, 矩阵运算库为 Eigen 3.3.3.

\section{1 纹理信息的保全}

保形参数化可以保证三维模型到平面模型的 映射不产生角度畸变, 但是会产生较大的面积畸 变, 这使得在通过仿射变换矩阵对纹理进行重映 射时会丢失较多的图像信息.

如图 7 所示, 对仅有保形参数化的花瓶模型进 行纹理重整, 将生成的纹理重新贴回到三维模型 上会出现很多模糊区域, 这便是保形参数化在纹 理重整中导致的信息丢失问题. 通过使用基于最 优传输的保测参数化, 可以最小化参数化过程中 引人的面积畸变, 保证纹理整合过程中信息的完 备性. 如图 8 所示, 将使用保测参数化产生的纹理 图片重新贴回到三维模型后, 之前丢失的细节得 到了保留。

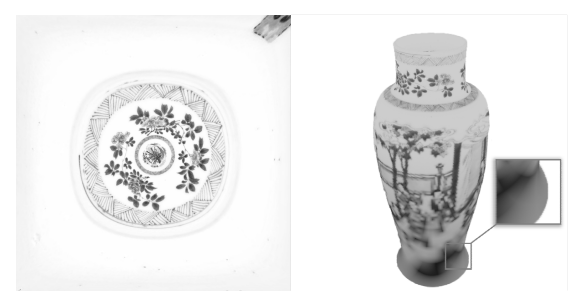

图 7 使用保形参数化的纹理重整

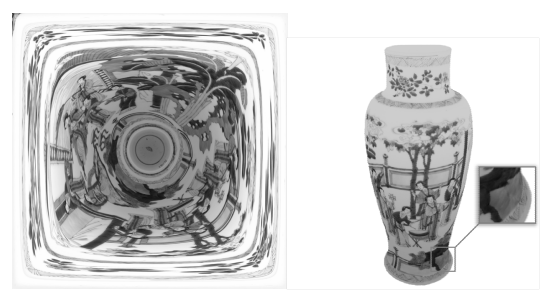

图 8 使用保测参数化的纹理重整 
为了更好地验证保测参数化的信息保全效果, 本文从 Sketchfab 上选择了不同种类与复杂度的扫 描模型进行实验并与文献[4]进行了比较，实验结 果如图 9 至图 15 所示, 其中从左至右分别为原始 纹理、原始纹理渲染图、本文方法重整纹理、本文 方法重整纹理的渲染图、文献[4]线框图、文献[4]
重整纹理的渲染图. 为了能够更清晰地展示文献[4] 优化图集的结果, 本文给出的是优化后图集的线框 图(简称文献[4]线框图). 从实验结果中可以看到, 重整后的纹理与模型的原始纹理以及经文献[4]优 化后的纹理在贴回三维模型后渲染的效果基本一致, 没有出现类似图 7 中花瓶底部大范围模糊的情况.
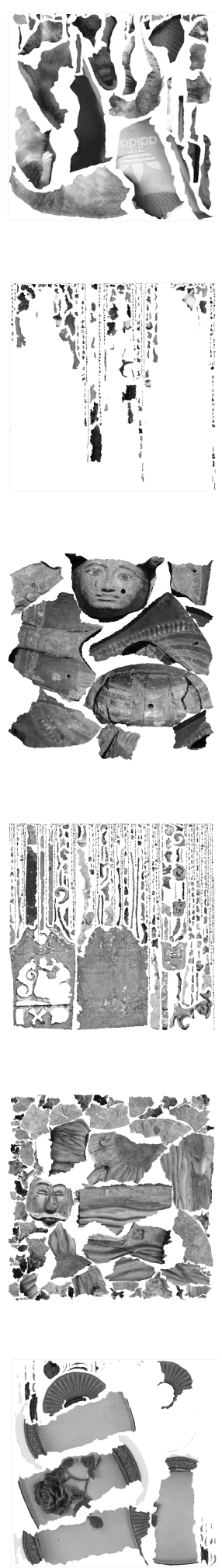
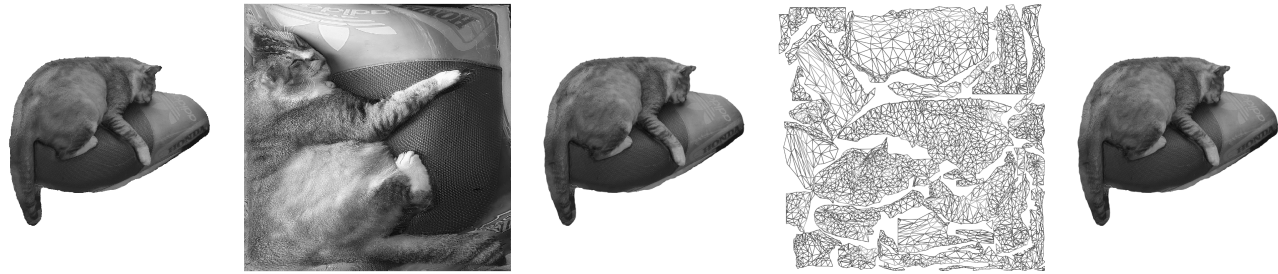

图 9 不同算法对猫咪 1 模型纹理整合实验结果
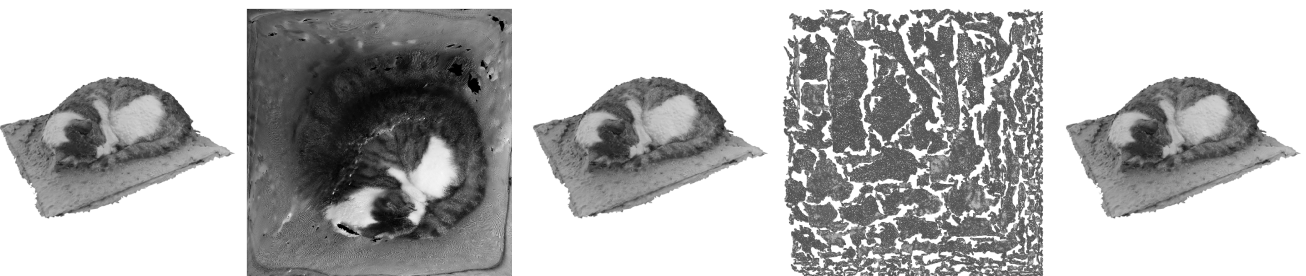

图 10 不同算法对猫咪 2 模型纹理整合实验结果
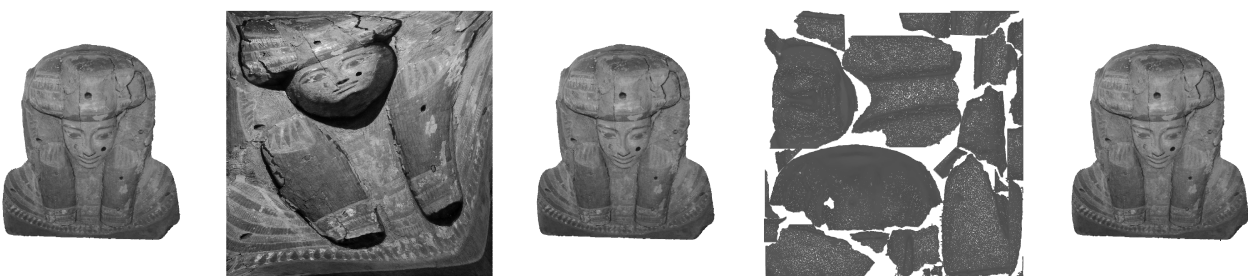

图 11 不同算法对狮身人面模型纹理整合实验结果
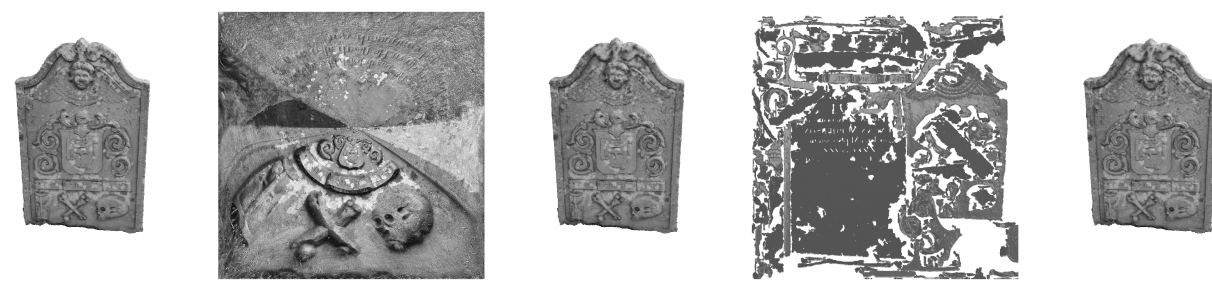

图 12 不同算法对墓碑模型纹理整合实验结果
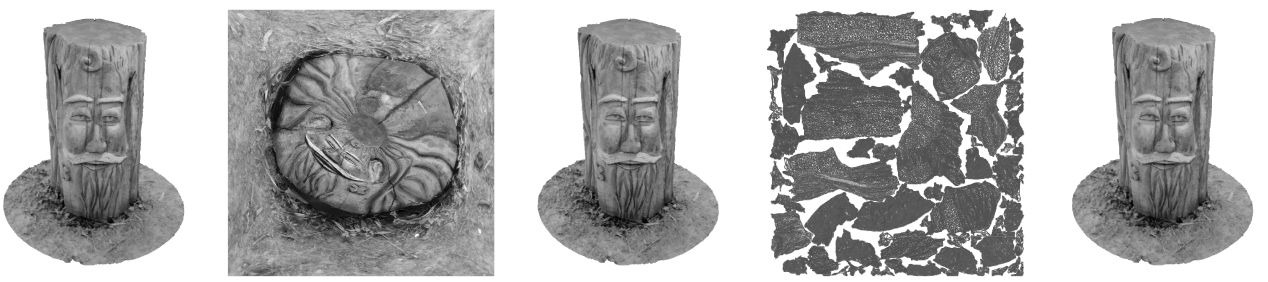

图 13 不同算法对树桩模型纹理整合实验结果
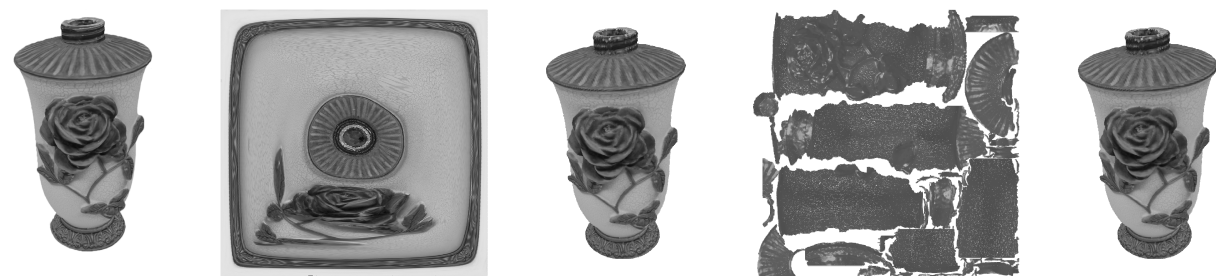

图 14 不同算法对花瓶 1 模型纹理整合实验结果 

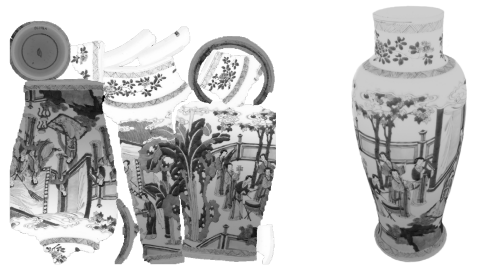

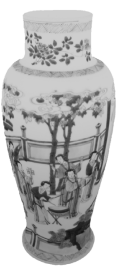

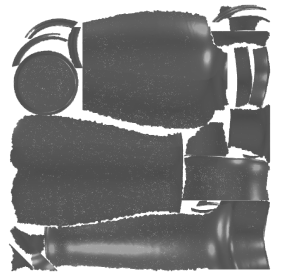

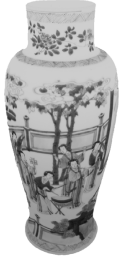

图 15 不同算法对花瓶 2 模型纹理整合实验结果

\section{2 纹理空间利用率}

纹理重整的关键目的是将碎片化的纹理重整 成一整块纹理，以提高图像的空间利用率. 本文统 计了原始纹理和重整纹理的空间利用率，并将其 结果与文献[4]进行了对比, 实验结果如表 1 所示. 从统计结果中可以看到, 当纹理碎片较少时, 文献 [4]的空间利用率较高, 但是随着碎片纹理的逐渐 增多，其纹理的空间利用率会逐渐降低. 而本文提 出的基于最优传输参数化的纹理重整算法既考虑 模型的面积畸变以及曲率高低, 也能够保证整合 后纹理的空间利用率达到 100.0\%.

表 1 纹理的空间利用率

\begin{tabular}{lccc}
\hline 模型名称 & 原始纹理 & 本文 & 文献[4] \\
\hline 猫咪 1 & 57.7 & 100.0 & 93.1 \\
猫咪 2 & 10.3 & 100.0 & 86.2 \\
狮身人面 & 71.7 & 100.0 & 94.2 \\
墓碑 & 46.4 & 100.0 & 84.7 \\
树桩 & 70.8 & 100.0 & 90.7 \\
花瓶 1 & 52.4 & 100.0 & 94.4 \\
花瓶 2 & 74.5 & 100.0 & 95.8 \\
\hline
\end{tabular}

\section{3 纹理映射的质量}

为了保证纹理图片在重新贴回到三维模型后 能够保留更多的细节, 且尽可能地不占用额外的 空间, 模型上三角形面元较大或曲率较高的区域 在纹理空间上对应的区域也应该越大，反之则越 小. 也就是说, 纹理上每处平均被采样次数的分布 应该是越均匀越好. 为了可视化重整后纹理的被 采样情况, 本文以模型中心为球心，在球面上均匀 地选取了 80 个位置点, 在这些点处放置摄像机对 模型进行渲染，并统计重整后纹理每个像素点被 采样的次数. 最后将被采样次数归一化得到采样 率, 并绘制了相应的热力图, 实验结果如图 16 所 示. 从采样率分布的热力图中可以看到，除去某些 几何细节特别丰富的区域，重整后的纹理像素的 采样率整体上分布均匀.

针对上述过程中得到的渲染图像，本文以原 始纹理的渲染图为基准，分别使用本文算法得到 的纹理与使用文献[4]得到的纹理对原始模型进行

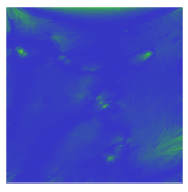

a. 猫咪 1

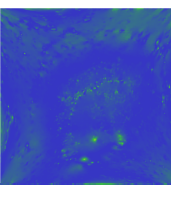

b. 猫咪 2

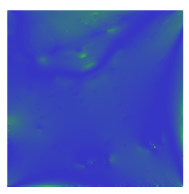

c. 狮身人面

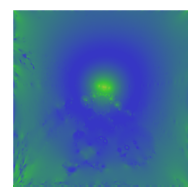

d. 墓碑

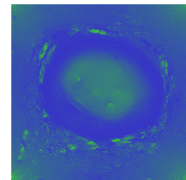

e. 树桩

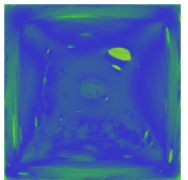

f. 花瓶 1

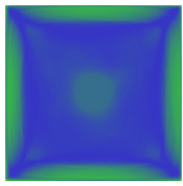

g. 花瓶 2
图 16 不同模型下的纹理映射采样率的热力图

渲染, 根据得到的图像计算其峰值信噪比 (peak signal to noise ratio, PSNR). 针对每个模型, 实验 记录其所有角度下渲染图 PSNR 的平均值, 结果如 表 2 所示. 通常, PSNR 的值越大, 代表着图像与原 始图像越接近, 重整纹理的质量也越好. 当 PSNR 的值低于 $20 \mathrm{~dB}$ 时, 说明图像的质量较差; 为 20 $30 \mathrm{~dB}$ 时, 说明图像质量一般; 为 $30 \sim 40 \mathrm{~dB}$ 时, 说 明图像整体质量较好; 而在 $40 \mathrm{~dB}$ 以上时, 说明图 像质量极好, 非常接近原始图像. 从实验结果中可 以看到, 使用本文算法得到的渲染图像整体质量 较好, 平均 PSNR 的值大多在 $35 \mathrm{~dB}$ 以上, 结果略 高于文献[4].

表 2 不同算法纹理渲染图像的平均 PSNR dB

\begin{tabular}{lcc}
\hline 模型名称 & 本文 & 文献[4] \\
\hline 猫咪 1 & 35.94 & 35.21 \\
猫咪 2 & 37.31 & 36.85 \\
狮身人面 & 36.29 & 35.85 \\
墓碑 & 32.80 & 32.21 \\
树桩 & 35.15 & 34.56 \\
花瓶 1 & 35.27 & 35.83 \\
花瓶 2 & 34.89 & 34.75 \\
\hline
\end{tabular}

上述的实验说明，尽管重整后的纹理经过了 一系列的变换, 图像会产生拉伸变形, 但是其最终 的渲染结果与使用原始纹理渲染得到的结果仍然 保持一致. 这进一步说明经过本文算法重整后的 纹理在保证纹理空间利用率为 $100 \%$ 的同时, 拥有 较高的质量. 


\section{6 结 语}

本文提出了一种基于离散最优传输的纹理重 整算法, 算法包括 3 个主要步骤: 输人待处理模 型, 对于亏格大于 0 的模型, 使用火烧法进行裁剪 预处理, 然后计算该模型的保测参数化映射, 最后 对纹理空间进行重整将碎片化的小纹理重整为一 个大纹理. 本文算法实现简洁且纹理重整的效果 较好, 可以在保证渲染质量的同时有效提高纹理 的空间利用率，对于高效压缩大规模模型的纹理图 像提供了一个有效的途径. 本文算法使用的保测参 数化在理论上保证了纹理在整个重整过程中信息的 完备性. 然而, 算法目前针对非流形网格以及高亏 格网格纹理的重整效果不够好, 存在由于割迹过长 导致的纹理畸变, 这也是本文下一步的工作重点.

\section{参考文献(References):}

[1] Prada F, Kazhdan M, Chuang M, et al. Gradient-domain processing within a texture atlas[J]. ACM Transactions on Graphics, 2018, 37(4): Article No.154

[2] Balmelli L, Taubin G, Bernardini F. Space optimized texture maps[J]. Computer Graphics Forum, 2002, 21(3): 411-420

[3] Dai Xuefeng, Xiong Hanjiang, Gong Jianya. A multi-texture automatic merging approach for the 3D city models[J]. Geomatics and Information Science of Wuhan University, 2015, 40(3): 347-352+411(in Chinese)

(戴雪峰, 熊汉江, 龚健雅.一种三维城市模型多纹理自动 合并方法 [J]. 武汉大学学报：信息科学版, 2015, 40(3): 347-352+411)

[4] Liu H Y, Fu X M, Ye C Y, et al. Atlas refinement with bounded packing efficiency[J]. ACM Transactions on Graphics, 2019, 38(4): Article No. 33

[5] Limper M, Vining N, Sheffer A. Box cutter: atlas refinement for efficient packing via void elimination[J]. ACM Transactions on Graphics, 2018, 37(4): Article No.153
[6] Garey M R, Johnson D S. Computers and intractability[M]. San Francisco: Freeman, 1979

[7] Kantorovich L V. On a problem of monge[J]. Journal of Mathematical Sciences, 2006, 133(4): 1383

[8] Brenier Y. Polar factorization and monotone rearrangement of vector valued functions[J]. Communications on Pure and Applied Mathematics, 1991, 44(4): 375-417

[9] Villani C. Optimal transport: old and new[M]. Heidelberg: Springer, 2008

[10] Zhou Yuming, Su Kehua. A GPU rendering algorithm for discrete optimal mass transportation[J]. Journal of Computer Aided Design \& Computer Graphics, 2019, 31(5): 726-735(in Chinese)

(周宇明, 苏科华. 使用 GPU 渲染的离散最优传输算法 [J]. 计算机辅助设计与图形学学报, 2019, 31(5): 726-735)

[11] Zhao X, Su Z Y, Gu X F, et al. Area-preservation mapping using optimal mass transport[J]. IEEE Transactions on Visualization and Computer Graphics, 2013, 19(12): 2838-2847

[12] Su K H, Chen W, Lei N, et al. Volume preserving mesh parameterization based on optimal mass transportation[J]. Computer Aided Design, 2017, 82: 42-56

[13] Franklin W R. Pnpoly-point inclusion in polygon test[OL]. [2020-09-07]. https://wrf.ecse.rpi.edu//Research/Short_Notes/ pnpoly.html

[14] Gu X F, Gortler S J, Hoppe H. Geometry images[C] //Proceedings of the 29th Annual Conference on Computer Graphics and Interactive Techniques. New York: ACM Press, 2002: 355-361

[15] Haker S, Angenent S, Tannenbaum A, et al. Conformal surface parameterization for texture mapping[J]. IEEE Transactions on Visualization and Computer Graphics, 2000, 6(2): 181-189

[16] Hormann K, Polthier K, Sheffer A, et al. Mesh parameterization: Theory and practice[C] //Proceedings of the ACM SIGGRAPH Asia 2008 Courses. New York: ACM Press, 2008: Article No.12

[17] Jin M, Kim J, Luo F, et al. Discrete surface Ricci flow[J]. IEEE Transactions on Visualization and Computer Graphics, 2008, 14(5): 1030-1043

[18] Su K H, Li C C, Zhou Y M, et al. Discrete Calabi flow: a unified conformal parameterization method[J]. Computer Graphics Forum, 2019, 38(7): 707-720 